\title{
Aproximações teóricas entre Pensamento Computacional e Abstração Reflexionante
}

Kátia Coelho da Rocha - PPGIE/UFRGS - katiacoelhorocha@gmail.com Marcus Vinicius de Azevedo Basso - PPGIE/UFRGS - mbasso@ufrgs.br Márcia Rodrigues Notare - PPGIE/UFRGS - marcia.notare@gmail.com

\section{Resumo}

O termo Pensamento Computacional ganhou destaque quando foi disseminado por Wing em 2006, desde lá sua definição vem sofrendo mudanças e críticas de pesquisadores da área da educação e da Ciência da Computação. A amplitude da definição permite diversas práticas e interpretações. Nós identificamos possíveis aproximações do Pensamento Computacional com a proposta dos "objetos-de-pensar-com" de Papert, com foco principal na abstração reflexionante de Piaget. Apresentamos uma discussão a respeito dessa aproximação, buscando apontar para a necessidade de práticas voltadas para o uso do Pensamento Computacional como uma possibilidade de contribuir para os processos de abstração reflexionante.

Palavras-chave: pensamento computacional, objetos-de-pensar-com, pensamento por procedimentos, abstração reflexionante

\section{Theoretical approaches between Computational Thinking and reflecting abstraction}

\begin{abstract}
The term Computational Thinking gained prominence when it was coined by Wing in 2006, since then its definition has undergone changes and criticism from researchers in the field of education and Computer Science. The breadth of the definition allows for different practices and interpretations. We identified possible approaches to Computational Thinking with Papert's proposal of "object-to-think-with", with a primary focus on Piaget's reflective abstraction. We present a discussion about this approach, seeking to point to the need for practices focused on the use of Computational Thinking as a possibility to contribute to the processes of reflective abstraction.
\end{abstract}

Key-words: computational thinking, object-to-think-with, procedural thinking, reflecting abstraction

\section{INTRODUÇÃO}

Este artigo compreende parte de uma pesquisa de doutorado sobre a temática Pensamento Computacional em processo de formação de professores. A pesquisa teve motivação a partir de investigações dos autores sobre contribuições da programação para o ensino de Matemática (Rocha, 2017). Atualmente, o termo Pensamento Computacional está em alta devido aos incentivos de empresas para a disseminação da computação, como consequência da crescente necessidade de profissionais na área, além da presença do termo em documentos oficiais que são fontes de referência para os currículos escolares.

Diante deste cenário, buscamos apresentar a definição de Pensamento Computacional que vem passando por diversas adaptações elaboradas por pesquisadores de diferentes áreas. Para compor esse quadro de definições partimos da leitura de publicações na área da ciência da computação e da informática na educação, tendo como base artigos publicados por Jeannette Wing, anais de dois workshops promovidos para debater sobre o Pensamento Computacional, além de documentos da International 
Society for Technology in Education (ISTE) e da American Computer Science Teachers Association (CSTA) e da Sociedade Brasileira de Computação (SBC) que fazem uma discussão a respeito do tema. Além disso, artigos publicados pela Association for Computing Machinery (ACM) e publicações nos anais do Congresso Brasileiro de Informática na Educação (CBIE) contribuíram para compor essa discussão a respeito da definição do termo Pensamento Computacional.

Com base na definição de Pensamento Computacional e toda a discussão que envolve a presença desse pensamento na prática escolar, que nem sempre está articulado com possíveis contribuições para os processos cognitivos, buscamos aproximações com as propostas de Papert e seus objetos-de-pensar-com e com a abstração reflexionante de Piaget.

Ao final do artigo propomos uma nova abordagem para uma atividade proposta em um livro didático sob o enfoque do Pensamento Computacional.

\section{AS DEFINIÇÕES DE PENSAMENTO COMPUTACIONAL}

O termo Pensamento Computacional, foi disseminado por Jeannette Wing, pesquisadora e professora da área da computação. Em 2006 Wing publicou o artigo, Computational Thinking em uma revista muito influente da computação, a Communications of the ACM, em que define Pensamento Computacional como um conjunto de habilidades da Ciência da Computação, presentes em diferentes áreas do conhecimento, e que podem trazer benefícios para todos, independentemente de serem ou não da área da computação (Wing, 2006). A autora defende que desde a infância todos deveriam ser estimulados a pensar como um cientista da computação, deixando claro que isso não se resume a programação, mas pensar em diferentes níveis de abstração (Wing, 2006; 2008). O termo abstração será definido na próxima seção seguindo a teoria de Piaget.

À medida que a ideia de Pensamento Computacional foi sendo aceita e discutida em diferentes níveis a definição de Wing também foi se tornando mais clara. Em 2010 a autora define como "os processos de pensamento envolvidos na formulação de problemas e suas soluções, para que as soluções sejam representadas de uma forma que possam ser efetivamente executadas por um agente de processamento de informações" (Wing, 2010, p. 1). Já em 2014, traz mais detalhes à definição: "O Pensamento Computacional é o processo de pensamento envolvido na formulação de um problema e na expressão de sua(s) solução(s) de forma que um computador - humano ou máquina - possa efetivamente executar" (Wing, 2014, p. 1).

Observa-se que a definição mais atual de Wing mostra que há processos mentais envolvidos durante a solução e a formulação de problemas e a forma de expressá-las pode ser feita entre humanos-humanos e/ou humanos-máquina, destacando que este pensamento não se resume a uma linguagem de programação, mas sim a uma forma de comunicação linguística (linguagem visual, natural, linguagem de programação, ...) que seja compreendida e esteja em um formato acessível ao público que se destina.

Analisemos agora outras definições e discussões a respeito da definição de Wing sobre Pensamento Computacional. A partir da publicação de Wing em 2006 o termo Pensamento Computacional ganhou espaço em diversas pesquisas na área, mas carecia de uma definição mais precisa. Neste sentido, foram propostos dois workshops promovidos pela National Academy of Sciences dos Estados Unidos da América, um em 2009 e outro em 2011. O primeiro workshop tinha como objetivo o escopo e a natureza do Pensamento Computacional. Diversos pesquisadores participaram e trouxeram diferentes visões sobre Pensamento Computacional, relacionando-o, entre outros, com: 
processos de pensamento procedural de Seymour Papert, inclusão de inteligência artificial, foco em processos e fenômenos abstratos, habilidade intelectual comparada à leitura e escrita, ferramenta para explicar e representar a complexidade (National Research Council, 2010). Ao final do workshop os participantes não entraram em consenso sobre os elementos e a estrutura do Pensamento Computacional e indicaram que isso pode ser um reflexo de que o "pensamento computacional, como modo de pensamento, tem seu próprio caráter distintivo" (National Research Council, 2010, p. 65). Já no workshop de 2011 o foco eram as dimensões cognitivas e educacionais, sendo apresentados exemplos que poderiam servir como indicadores de como as pessoas veem a intersecção da computação com conhecimentos das diversas áreas e algoritmos (National Research Council, 2011).

Na tentativa de tornar o conceito de Pensamento Computacional mais acessível aos educadores a ISTE e a CSTA reuniram empresas e professores de vários níveis para criar uma definição operacional para o termo. Essa definição apresenta que:

\begin{abstract}
O pensamento computacional é um processo de resolução de problema, com as seguintes características: formulação de problemas de uma forma que permita usar um computador e outras ferramentas para ajudar a resolvê-los; organização lógica e análise de dados; representação de dados por meio de abstrações como modelos e simulações; automação de soluções por meio do pensamento algorítmico (a série de passos ordenados); identificação, análise e implementação de soluções possíveis com o objetivo de alcançar a mais eficiente e efetiva combinação de etapas e recursos; e generalização e transferência desse processo de resolução de problemas para uma ampla variedade de problemas. (ISTE/CSTA, 2011, p. 7, tradução nossa)
\end{abstract}

Além desta definição operacional, a ISTE/CSTA descreve um conjunto de disposições e atitudes que o estudante deve apresentar para desenvolver o Pensamento Computacional, são elas:

confiança em lidar com complexidade; persistência em trabalhar com problemas difíceis; tolerância para ambiguidades; habilidade de lidar com problemas abertos; habilidade de comunicar e trabalhar com outros para atingir um objetivo ou uma solução em comum (ISTE/CSTA, 2011, p.7, tradução nossa)

A ISTE/CSTA ainda apresenta nove conceitos a serem abordados nos currículos escolares: coleta de dados, análise de dados, representação de dados, decomposição de problema, abstração, algoritmos, automação, simulação e paralelização. Nota-se que essa definição possui uma extensa lista de características, mais uma lista de atitudes e conceitos a serem desenvolvidos, na tentativa de aproximar dos professores acaba também ampliando essa visão e misturando-a a outras habilidades fundamentais que se espera do estudante.

Observando a realidade brasileira identificamos que iniciativas de desenvolvimento do Pensamento Computacional e pesquisas na área são apresentados no Workshop de Ensino em Pensamento Computacional, Algoritmos e Programação e outros workshops do CBIE. Além disso, existe uma tentativa da SBC em inserir no currículo eixos relacionados à computação: Cultura Digital, Mundo Digital e Pensamento Computacional. A SBC define Pensamento Computacional como a "Habilidade de compreender, definir, modelar, comparar, solucionar, automatizar e analisar problemas (e soluções) de forma metódica e sistemática" (SBC, 2019, p. 2). Essa definição da SBC e sua proposta curricular não consta nos programas curriculares oficiais, ela é uma proposta dessa sociedade para as escolas que têm interesse em incluir a Computação na Educação Básica. 
Abaixo apresentamos a tabela 1 que expressa a evolução do conceito de Pensamento Computacional com o passar do tempo, relacionando-o com autores/instituições e palavras-chaves que destacamos de cada definição.

Tabela 1 - Evolução do conceito de Pensamento Computacional

\begin{tabular}{lll}
\hline Ano & Autor/Instituição & Palavras-chave \\
\hline 2006 & Wing & Habilidades mentais, abstração \\
2009 & Workshop NRC & $\begin{array}{l}\text { processos de pensamento por procedimentos, inteligência artificial, } \\
\text { processos e fenômenos abstratos, habilidade intelectual, ferramenta para } \\
\text { explicar e representar a complexidade }\end{array}$ \\
2010 & Wing & $\begin{array}{l}\text { processos de pensamento, formulação e resolução de problemas, } \\
\text { representação }\end{array}$ \\
2011 & ISTE/CSTA & $\begin{array}{l}\text { processos de resolução e formulação de problemas, diversas ferramentas, } \\
\text { representação, pensamento algorítmico, análise, automação, simulação, } \\
\text { generalização, transferência para outros problemas }\end{array}$ \\
2014 & Wing & $\begin{array}{l}\text { processos de pensamento, formulação e resolução de problemas, } \\
\text { abstração, comunicação linguística }\end{array}$ \\
& & SBC
\end{tabular}

As definições apresentadas acima nos mostram o quanto o conceito de Pensamento Computacional ainda está em construção, e a própria Wing mostra suas evoluções a respeito da temática. Ainda com essas evoluções é possível identificar que no geral todas elas procuram associar ao termo a resolução de problemas e alguma forma de comunicar seja por meio de algoritmos ou outras formas de representação.

$\mathrm{Hu}$ (2011), publicou um artigo em que apresenta diferentes interpretações para a compreensão de Pensamento Computacional, pois segundo ele, até o momento da publicação do seu artigo, as definições não deixavam claro quais seriam os elementos, estruturas ou peculiaridades do Pensamento Computacional. $\mathrm{O}$ autor sugere que o Pensamento Computacional pode ser uma mistura de elementos de raciocínio vindos de vários outros raciocínios como lógico, matemático, analítico. Defende a necessidade de desenvolver um pensamento crítico nos diversos níveis integrado ao ensino e o Pensamento Computacional pode ser uma forma de auxiliar esse processo.

Identificamos práticas que estão voltadas estritamente ao ato de programar (Oliveira e Marquiori, 2019; Nunes e Kologeski, 2019; Pascual Jr. e Oliveira, 2019), como uma forma de promover o Pensamento Computacional. De acordo com Voogt et al. (2015) essas abordagens se devem ao fato de estudos anteriores usarem a programação como uma forma de desenvolver habilidades de pensamento. Porém, o ato de programar não indica que essas habilidades serão transferidas para outros domínios (Pea; Kurland, 1984). A própria Wing admite que pensar como cientista da computação vai além de programar um computador, mas pensar em vários níveis de abstração (Wing, 2006).

Denning (2017) faz um resgate do termo Pensamento Computacional que já era usado na Ciência da Computação há mais de 60 anos, com enfoques diferentes do atual. $\mathrm{O}$ autor demonstra preocupação com a forma como o Pensamento Computacional tem ocupado os espaços escolares: um certo exagero onde tudo é Pensamento Computacional, programar é essencial e descrever qualquer ação é um algoritmo. Assim, ele argumenta que deveríamos olhar para a definição de Aho,

Abstrações chamadas de modelos computacionais estão no centro da computação e do pensamento computacional. A computação é um processo definido em termos de um 
modelo subjacente de computação e o pensamento computacional são os processos de pensamento envolvidos na formulação de problemas para que suas soluções possam ser representadas como etapas computacionais e algoritmos. (Aho, 2011, p. 7)

A definição de Aho resgata a essência de 60 anos da ciência da computação, além de trazer o Pensamento Computacional para outros campos, já que o foco está nos processos mentais envolvidos na formulação dos problemas. A própria Wing (2014) admite as contribuições de Aho em sua definição mais atual, acrescentando a importância dos processos mentais, além de valorizar os modelos que possam ser efetivos no meio computacional ou ainda solucionáveis por humanos.

Essa última definição de Wing, associada a Aho, mostra as possibilidades do Pensamento Computacional dentro de uma perspectiva do "fazer computacional" em que as pessoas possam aprender em suas áreas, tornando-se melhores por meio da computação (Denning, 2017; Hemmendinger, 2010).

A perspectiva do Pensamento Computacional mais voltado aos processos mentais que podem ser aplicados em diferentes domínios nos remete às ideias propostas por Seymour Papert na década de 80. Essas ideias serão discutidas na próxima seção e articuladas com os processos de abstração propostos por Piaget.

\section{PENSAMENTO COMPUTACIONAL: OS OBJETOS-DE-PENSAR-COM E OS PROCESSOS DE ABSTRAÇÃO REFLEXIONANTE}

No final da década de 60 Seymour Papert e sua equipe do Massachusetts Institute of Technology (MIT) criaram o LOGO, uma linguagem de programação pensada para crianças com o intuito de que elas pudessem aprender a programar de uma forma mais simples, de acordo com sua realidade.

Além de criar a linguagem Papert traz uma reflexão a respeito dos efeitos do LOGO e os impactos que a tecnologia computacional pode trazer para as crianças, criando "novas possibilidades de aprender, pensar e crescer tanto emocional como cognitivamente" (Papert, 1985, p. 34).

Na década de 80 em seu livro Logo: Computadores e Educação, Papert já usava o termo Pensamento Computacional, relacionando-o com as habilidades mentais desenvolvidas nos processos de programação. No ambiente LOGO o sujeito programa o computador, ou seja, "ensina o computador" e ao fazer isso ele explora a forma como pensa, o que o torna um epistemólogo, ampliando o poder de reflexão sobre o seu próprio pensamento. Neste sentido o computador torna-se um "objeto-de-pensar-com", um instrumento que é fruto de uma interseção de presença cultural, conhecimento implícito e identificação pessoal (Papert, 1985).

Os objetos-de-pensar-com tornam-se instrumentos que o sujeito pode se apropriar para auxiliá-lo em seu processo de pensar sobre diferentes conceitos. Ao programar a tartaruga do LOGO, por exemplo, ela é utilizada como um objeto-de-pensar-com, que permite ao sujeito usar uma linguagem computacional para se comunicar ao mesmo tempo em que reflete sobre os conceitos implícitos na atividade. Assim, Papert sugere que "pensemos como um computador", ou seja, pensemos por procedimentos. Ao pensar por procedimentos o sujeito precisa descrever o processo com precisão de forma que possa ser executado pela máquina. Quando escreve um procedimento, suas intuições tornam-se mais evidentes, permitindo a reflexão e aproximando-o do conhecimento formal.

Papert afirma que diariamente somos levados a pensar como um computador, mas nem sempre esses pensamentos são objetos de reflexão. Ele sugere que pensemos como 
um computador em situações diárias, refletindo sobre "procedimentos para pessoas", explorando o poder intelectual dessa ferramenta.

Ao propor o pensamento por procedimentos como um objeto-de-pensar-com Papert traz um novo e potente uso do computador para a época. Ele vislumbra a importância desse tipo de pensamento, um processo de descrição analítico que exige um certo nível de precisão que deve ser executado por uma máquina ou ainda pode ser ampliado para pessoas.

As ideias de Wing de Pensamento Computacional não fazem alusão às ideias de Papert, mas podemos encontrar aproximações no fato de identificar os benefícios do pensamento por procedimentos. Em suas definições de Pensamento Computacional Wing $(2006$; 2010; 2014) destaca a abstração como o elemento mais importante desse pensamento. Segundo a autora o processo de abstração implica em decidir quais detalhes precisam ser destacados e quais podem ser ignorados (Wing, 2006; 2014). Esse processo de abstração compreende, conforme expressam Ribeiro et al (2020):

- abstrações para representar informações - é necessário criar abstrações adequadas para os diversos tipos de dados que podem estar contidos no algoritmo. Os dados podem ser números, pilhas de provas, um mapa, entre outros e cada um deles vai exigir uma representação. Por exemplo para descrever um algoritmo que representa uma rota em um mapa é necessário que esse mapa seja visto como um conjunto de cidades ligadas por estradas. Escrever algoritmos exige do usuário visualizar dados como uma composição de dados mais simples. Desta forma, é possível usar vários níveis de abstração para resolver um problema.

- abstrações para descrever algoritmos - é necessário selecionar as instruções (relacionase à linguagem utilizada) e quais as operações (composição, escolha, repetição) podem ser utilizadas para descrever os procedimentos.

- técnicas para construir algoritmos - além dos processos anteriores de abstração são necessárias técnicas para escrever os algoritmos que permitem percorrer o caminho do enunciado à solução do problema com mais facilidade. Essas técnicas também exigem abstrações do sujeito. Entre elas podemos destacar: decomposição (decompor o problema em partes e definir interfaces de cada subproblema), generalização (reutilizar e adaptar algoritmos) e transformação (utilizar uma solução para outros problemas através de transformações como reuso, refinamento, evolução, redução, ...)

A partir dos exemplos acima podemos identificar que para a Ciência da Computação um algoritmo é um exemplo de abstração que pode ocorrer em diferentes níveis. E a abstração é um mecanismo que auxilia na captura das propriedades comuns nos conjuntos de objetos (Wing, 2014). Este é o ponto que queremos essencialmente abordar nessa teoria em comparação com as ideias de Papert e Piaget.

O significado atribuído por Wing à abstração está relacionado ao lado operacional de criar uma solução para um determinado problema. Papert, baseado em Piaget, quando trata das possibilidades do pensamento por procedimentos está pensando não só nessa abstração, mas nos efeitos e contribuições que ela pode trazer ao modo de pensar, pensando em uma abstração no sentido piagetiano. Vejamos o que Piaget propõe sobre abstração.

Piaget define que existem basicamente dois tipos de abstração: a abstração empírica e a abstração reflexionante. A abstração empírica está relacionada com as informações físicas do objeto, enquanto a abstração reflexionante envolve níveis mais elevados de cognição, pois está relacionada às coordenações de ações do sujeito (Montangero; Naville, 1998). A abstração reflexionante provém de características não observáveis dos objetos, mas das atividades cognitivas do sujeito (esquemas, 
coordenações, operações, estruturas, ...) que são retiradas e utilizadas em outros problemas, situações, adaptações (Piaget, [1977],1995).

Ao definir a abstração reflexionante, Piaget apresenta que ela envolve dois processos: o reflexionamento que é a projeção sobre um patamar mais elevado que foi retirado de um inferior; e a reflexão um ato mental que envolve a reconstrução e reorganização sobre o patamar superior que foi transferido de um inferior (Becker, 2012). Quando o resultado de uma abstração reflexionante envolve tomada de consciência ela é chamada de abstração refletida.

Há ainda um outro tipo de abstração reflexionante, a abstração pseudo-empírica quando o objeto é modificado pela ação do sujeito e enriquecido de propriedades retiradas das suas coordenações, ou seja, a propriedade abstraída não é do objeto.

Ao comparar as definições de abstração podemos identificar que o termo usado por Wing, em Piaget, contempla processos de abstrações pseudo-empíricas e abstrações reflexionantes. Wing menciona em seus artigos (Wing, 2006; 2010; 2014) que alguns benefícios podem ocorrer, mas não deixa claro como o Pensamento Computacional e consequentemente as sucessivas abstrações realizadas em processos computacionais poderiam contribuir para a aprendizagem.

Já Papert (1985) quando se refere à importância do uso dos objetos-de-pensarcom está relacionando-os como uma forma de suporte à reflexão, algo que permitiria ao sujeito pensar sobre seu próprio pensamento, estendendo essa forma de pensar para outras situações. Esse movimento está relacionado com os processos de abstração reflexionante, aperfeiçoando os mecanismos de reflexão do sujeito, ampliando suas ferramentas mentais (Papert, 1985).

Devido a esta imprecisão na definição, já apontada por Denning (2017) e a falta de uma análise mais detalhada sobre possíveis efeitos do Pensamento Computacional observamos que diversos tipos de práticas são difundidas e fazem uso desse termo como referência. Muitas dessas práticas estão mais engajadas em ensinar ou inserir computação no dia a dia, trazendo o ato de programar como chave do processo ou ainda propondo escritas de procedimentos sem valorizar as possíveis abstrações reflexionantes que essas práticas podem proporcionar. Ao resgatarmos a proposta dos objetos-de-pensar-com de Papert acrescentamos o conceito de abstração reflexionante ao conceito do Pensamento Computacional. Assim, o foco principal, na nossa visão de Pensamento Computacional, está nos processos mentais que englobam elementos da computação e que podem servir como objetos-de-pensar-com.

\section{UM EXEMPLO DE PENSAMENTO COMPUTACIONAL}

Nesta seção nos propomos a apresentar uma possibilidade de aplicação do Pensamento Computacional mais voltado ao olhar de um objeto-de-pensar-com. Para isso selecionamentos uma atividade comumente apresentada em livros didáticos e que poderia ser remodelada para que pudesse estar mais voltada ao desenvolvimento do Pensamento Computacional.

A atividade escolhida está apresentada em um livro didático (Editora Moderna, 2010) de sexto ano do Ensino Fundamental, em que propõe que o aluno descubra o peso do mamão. Essa atividade está inserida no capítulo do livro que trata das operações de adição e subtração com números naturais. Observe a atividade ilustrada pela figura do quadro 2.

Nossa proposta consiste em remodelar essa atividade, para isso sugerimos que seja proposto ao sujeito descrever um procedimento que expresse o método para calcular 
o peso do mamão. No quadro abaixo apresentamos dois possíveis procedimentos que podem expressar o cálculo do peso do mamão.

Quadro 2: Atividade com balanças - procedimentos possíveis para a solução do problema

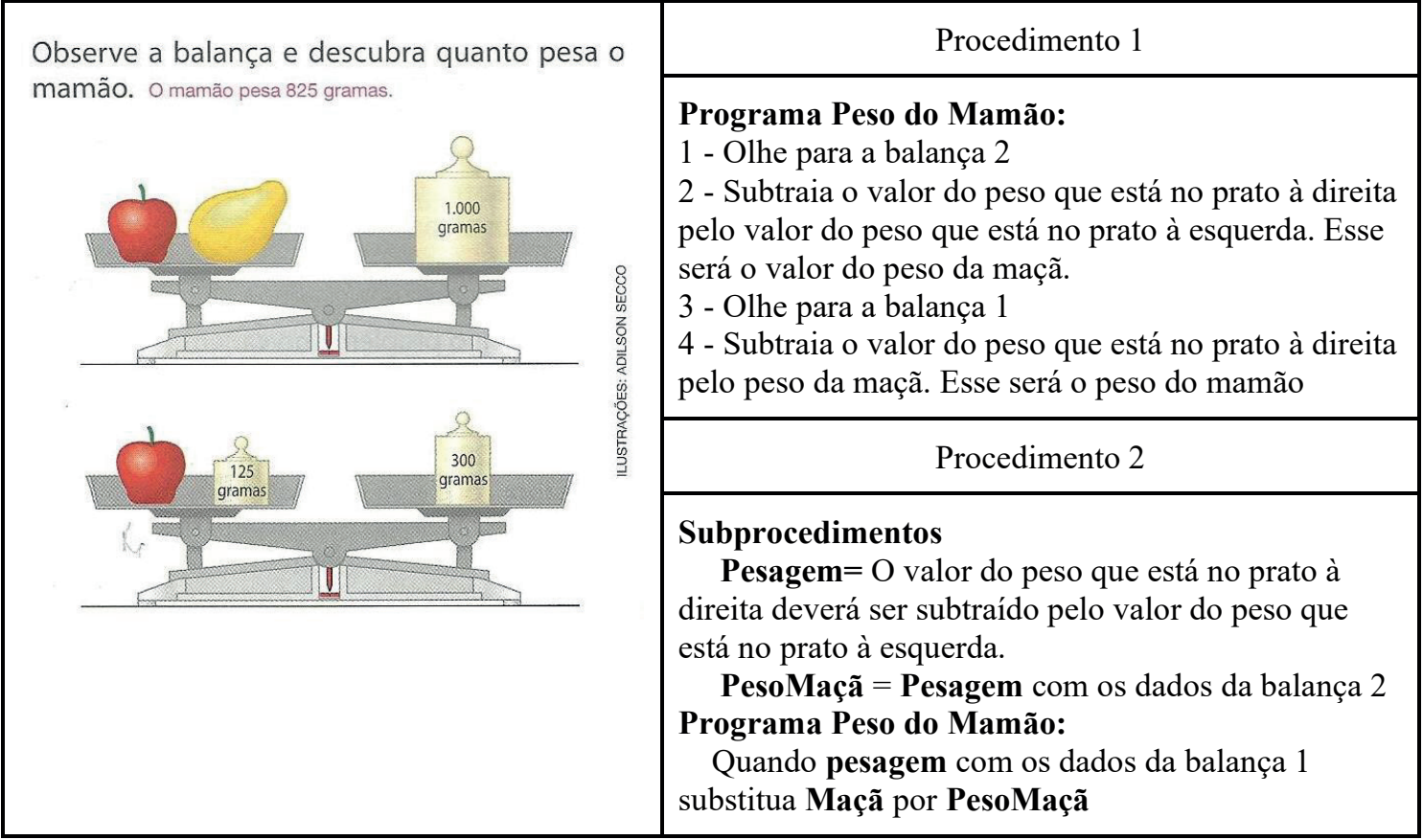

O procedimento 1 descrito acima representa um programa linear mais semelhante a uma instrução. O procedimento 2 traz um programa separado em subprocedimentos dividindo o processo em etapas que podem ser olhadas separadamente e que são combinadas para compor o procedimento final.

Descrever uma solução através de um procedimento não é uma tarefa simples e usual da escola, nem sempre os sujeitos são provocados a expressar como pensam de forma detalhada. Ao propor a solução através desse formato queremos provocar nos sujeitos os elementos apontados por Papert e também por Wing, pensar sobre o próprio pensamento. Do ponto de vista Piagetiano, observamos que os dois procedimentos podem provocar abstrações reflexionantes, mas o segundo traz elementos mais complexos, já que envolve um passo além da simples descrição, mas articulação de diversos esquemas, coordenações e estruturas elaboradas ou em fase de elaboração.

Papert (1985) afirma que um bom programa computacional e que também pode ser um bom modelo para auxiliar nos processos de reflexão é aquele que envolve uma programação estruturada, ou seja, que utiliza subprocedimentos, pois ele pode ser separado em etapas facilitando a compreensão e a correção de possíveis erros. Além disso, afirma que o uso da programação paralela (programas que são executados simultaneamente), como é caso do procedimento 2 , torna o processo mais claro e compreensível.

\section{CONSIDERAÇÕES FINAIS}

O termo Pensamento Computacional disseminado por Wing em 2006, apresenta uma evolução em sua definição com o passar do tempo. Essas diferentes definições essencialmente relacionam o Pensamento Computacional à resolução de problemas e a processos mentais. As ideias de Pensamento Computacional ainda apresentam certa imprecisão na visão de educadores e cientistas da computação (Denning, 2017) o que gera diferentes práticas associadas ao termo. As práticas têm enfoques variados: apenas programar, descrição de algoritmos, atividades com uso de tecnologia, aplicação de 
computação desplugada (atividades sem uso de tecnologias digitais), entre outras. O fato é que nem todas essas práticas observam com o devido cuidado o objetivo do desenvolvimento dos processos mentais.

Os processos mentais propostos por Wing e os diversos níveis de abstração que podem estar envolvidos com o Pensamento Computacional não são amplamente explorados nas publicações. Nós acreditamos que essas ideias podem ser aproximadas com as propostas dos objetos-de-pensar-com de Papert e com os processos de abstração reflexionante de Piaget.

Nesse sentido, propomos que práticas associadas ao Pensamento Computacional tenham como foco principal esses elementos. Com o intuito de contribuir para essa discussão, propomos uma atividade que poderia ser aplicada sem o uso do computador, mas que utiliza elementos da computação e que visa observar e contribuir com possíveis abstrações reflexionantes. Pretendemos estender essas ideias em atividades de formação de professores analisando as contribuições do Pensamento Computacional nos processos de cognição.

\section{REFERÊNCIAS BIBLIOGRÁFICAS}

AHO, A. Computation and Computational Thinking. In: Ubiquity Symposium, jan. 2011, ACM Ubiquity

BECKER, F. Educação e construção do conhecimento. 2 ed., Porto Alegre: Penso, 2012. 200p.

BRASIL. Base Nacional Comum Curricular (BNCC). Brasília: MEC, 2017. Disponível em: http://basenacionalcomum.mec.gov.br/images/BNCC_EI_EF_110518_versaofinal_site.pdf. Acesso em: 10 out. 2020.

BRASIL. Base Nacional Comum para a Formação Inicial de Professores da Educação Básica (Bnc-formação): MEC, 2019.

ISTE/CSTA. Computational Thinking Teacher Resource. 2 ed., 2011. Disponível em: www.iste.org/docs/ct-documents/ct-teacher-resources_2ed-pdf.pdf?sfvrsn=2. Acesso em: 20 out. 2020.

DENNING, P. Remaining Trouble Spots with Computational Thinking. Communications of the ACM, vol. 60, n. 3, p.33-39, mar 2017.

EDITORA MODERNA (org.). Projeto Araribá: matemática $-\mathbf{6}^{\mathbf{0}}$ ano. $3^{\mathrm{a}}$ Ed. Editor Responsável Fábio Martins de Leonardo. São Paulo: Moderna, 2010.

HEMMENDINGER, D. A please for modesty. ACM Inroads, vol.1, n. 2, p. 4-7, 2010

HU, C. Computational Thinking - What It Might Mean and What We Might Do About It. In: Proceedings of the 16th annual joint conference on Innovation and technology in computer science education, ITiCSE'11, 2011, New York: ACM, 2011 p. 223-227.

MARQUIORI, V. S.; OLIVEIRA, M. O Pensamento Computacional na Compreensão de Problemas do Cotidiano Feminino para o Letramento em Programação. Anais dos Workshops do VIII Congresso Brasileiro de Informática na Educação (WCBIE 2019). Disponível em https://www.br-ie.org/pub/index.php/wcbie/article/view/8992. Acesso em: 30 out. 2020.

MONTANGERO, J; MAURICE-NAVILIE, D. Piaget ou a Inteligência em evolução: Sinopse cronológica e Vocabulário. Tradução de Tânia Beatriz Iwaszko Marques e Fernando Becker. Porto Alegre: Artmed, 1998. 764 p 
NATIONAL RESEARCH COUNCIL (U.S.); COMMITTEE FOR THE WORKSHOPS ON COMPUTATIONAL THINKING. Report of a workshop on the scope and nature of computational thinking. Washington, D.C.: National Academies Press, 2010

NATIONAL RESEARCH COUNCIL (U.S.) (ORG.). Report of a workshop of Pedagogical Aspects of Computational Thinking. Washington, D.C: National Academies Press, 2011.

NUNES, N. B.; KOLOGESKI, A. L. Análise de iniciativas envolvendo lógica de programação para alunos de ensino médio. Anais dos Workshops do VIII Congresso Brasileiro de Informática na Educação (WCBIE 2019). Disponível em https://www.br-ie.org/pub/index.php/wcbie/article/view/8972. Acesso em: 30 out. 2020.

PASQUAL Jr, P. A.; OLIVEIRA, S. Pensamento computacional: uma proposta de oficina para a formação de professores Revista Novas Tecnologias na Educação. V. 17, n. 1, maio 2019. Disponível em https://seer.ufrgs.br/renote/article/view/95707. Acesso em: 30 out. 2020 .

PAPERT, S. Logo: computadores e educação. Tradução: José Armando Valente. São Paulo: Brasiliense, 1985. 253 p.

PEA, R. D.; KURLAND, D. M. On the cognitive effects of learning computer programming. New Ideas Psychol, vol. 2, n. 2, p. 137 -168, 1984.

PIAGET, J. Abstração reflexionante: Relações lógico-aritméticas e ordem das relações espaciais. Porto Alegre: Artes Médicas, [1977] 1995. 292 p.

RIBEIRO, L.; FOSS, L.; CAVALHEIRO, S. A. C. Entendendo o Pensamento Computacional. In: RAABE, A.; ZORZO, A. F.; BLIKSTEIN, P. (Org). Computação na Educação Básica: fundamentos e experiências. Porto Alegre: Penso, 2020. p.16-30.

ROCHA, K. C. Programação em Scratch na sala de aula de Matemática:

investigações sobre a construção do conceito de ângulo, no Estado do Rio Grande do Sul. Porto Alegre: PPGEMat/UFRGS, 2017. 211p. Dissertação de Mestrado.

SBC. Diretrizes para o ensino de Computação na Educação Básica. 2019. Disponível emhttps://www.sbc.org.br/educacao/diretrizes-para-ensino-de-computacao-naeducacao-basica. Acesso em 17 out. 2020

VOOGT, J.; FISSER, P.; GOOD, J.; MISHRA, P.; YADAV, A. Computational thinking in compulsory education: Towards an agenda for research and practice. Education and Information Technologies, vol. 20, p. 715-728, 2015.

WING, J. M. Computational Thinking. Communications of the ACM, vol. 49, n. 3, p.33-35, mar 2006.

WING, J. M. Computational thinking and thinking about computing. Philosophical Transactions of the Royal Society A: Mathematical, Physical and Engineering Sciences, v. 366, n. 1881, p. 3717-3725, 2008

WING, J. M. Computational Thinking: What and Why?, 17. out. 2010. Disponível em: http://www.cs.cmu.edu/ CompThink/resources/TheLinkWing.pdf. Acesso em: 15 out. 2020 .

WING, J. M. Computational Thinking Benefits Society. Social Issues In Computing, New York, 10 jan. 2014. Disponível em: http://socialissues.cs.toronto.edu/ 2014/01/computational-thinking/. Acesso em: 15 out. 2020. 